\title{
Erasing no-man's land by thermodynamically stabilizing the liquid-liquid transition in tetrahedral particles
}

\author{
Frank Smallenburg ${ }^{1 \star}$, Laura Filion ${ }^{2}$ and Francesco Sciortino ${ }^{1,3}$
}

One of the most controversial hypotheses for explaining the origin of the thermodynamic anomalies characterizing liquid water postulates the presence of a metastable second-order liquid-liquid critical point' located in the "no-man's land'2. In this scenario, two liquids with distinct local structure emerge near the critical temperature. Unfortunately, as spontaneous crystallization is rapid in this region, experimental support for this hypothesis relies on significant extrapolations, either from the metastable liquid or from amorphous solid water ${ }^{3,4}$. Although the liquid-liquid transition is expected to feature in many tetrahedrally coordinated liquids, including silicon ${ }^{5}$, carbon $^{6}$ and silica, even numerical studies of atomic and molecular models have been unable to conclusively prove the existence of this transition. Here we provide such evidence for a model in which it is possible to continuously tune the softness of the interparticle interaction and the flexibility of the bonds, the key ingredients controlling the existence of the critical point. We show that conditions exist where the full coexistence is thermodynamically stable with respect to crystallization. Our work offers a basis for designing colloidal analogues of water exhibiting liquid-liquid transitions in equilibrium, opening the way for experimental confirmation of the original hypothesis.

Numerous studies have attempted to provide evidence of a second critical point in water ${ }^{2,7-9}$, silicon ${ }^{10}$ and other tetrahedral liquids. Unfortunately, in all cases its expected location is well within the region where these liquids spontaneously crystallize and so no conclusive evidence has ever been reported. To observe critical fluctuations, the liquid must survive in its metastable supercooled state long enough to equilibrate, and the experimental observation time needs to be smaller than the spontaneous nucleation time. In principle, these conditions are easier to meet in simulation studies, in which heterogeneous nucleation is absent by construction and homogeneous nucleation is slowed down by the small size of the investigated sample. Despite these advantages, numerical evidence for the existence of a liquid-liquid critical point (LLCP) has been reported only for a small set of models ${ }^{1,11-16}$, and in some of the cases, not in a definitive way. It has even been speculated ${ }^{17}$ (but not confirmed by studies repeated by independent groups ${ }^{15,16,18}$ ) that the LLCP is a misinterpreted liquid-crystal transition, casting doubt on the relevance and appropriateness of this elegant hypothesis for interpreting the behaviour of water and silica, two of the most common substances on Earth.

If the liquid-liquid (LL) scenario has universal aspects, it should be possible to isolate its key ingredients in terms of the interparticle interactions to pinpoint the physical origin of the phenomenon. It should also be possible to clarify the contradictory results available in the literature and identify conditions in which the LLCP can be unambiguously observed in experiments. This requires a thorough investigation, not only of the liquid(s), but also of the relative stability of the disordered and ordered phases. Here, our investigation is inspired by two distinct recent theoretical discoveries: that network interpenetration can facilitate a LL transition ${ }^{19}$, and that bond directionality is crucial in controlling crystallization ${ }^{20}$. According to the first, particles with long arms and strong directional interactions can form fully bonded interpenetrating network structures separated by a first-order transition line. According to the second, when bonds are highly flexible, the liquid phase can remain stable in an intermediate range of densities down to very low temperatures. If the bond flexibility destabilizes the crystal state more rapidly than the LL transition, it might be possible to observe a genuine phase separation into two thermodynamically stable liquid phases, with no interference of crystallization at any temperature $T$.

Guided by the previously described discoveries, and mimicking the DNA tetravalent nanostars that have recently been experimentally investigated ${ }^{21-23}$, we develop a simple limitedvalence model in which bond length and bond flexibility can be continuously varied. We attach to a central spherical core of diameter $\sigma$ four identical spheres, generating four arms arranged in a tetrahedral geometry as depicted in Fig. 1. The centres of the four arms are located a distance $L$ from the centre of the core sphere. The position of each of the arms in a tetramer is not fixed, but rather can deviate from its 'ideal' tetrahedral position by a maximum angle $\phi$ at no energetic cost, thus simply modelling arm flexibility. An attractive patch is located on each of the four arms, pointing directly away from the centre of the core. The patches interact through the commonly used angular square-well (of depth $\epsilon$ ) Kern-Frenkel potential ${ }^{24}$ (Methods), with parameters (the angular width $\cos \theta$ and the interaction range $\delta$ ) satisfying the single bond per patch condition. When $L=0$ and $\phi=0$, the model reduces to a sphere of diameter $\sigma$ with four tetrahedrally oriented patches; for this case, previous studies have shown that the liquid crystallizes on cooling, without any evidence of a LLCP (ref. 25). In summary, the angle $\phi$ increases the bond flexibility beyond $\theta$ and $L$ controls the effective 'softness' of the interaction. In this study we investigate more than 20 models, generated by different combinations of $L$ and $\cos \phi$. For each of these, we examine (using successive umbrella sampling (SUS) grand-canonical simulations ${ }^{26}$ ) approximately five to ten different $T$ and two

${ }^{1}$ Department of Physics, Sapienza, Universitá di Roma, Piazzale Aldo Moro 2, I-00185 Roma, Italy, ${ }^{2}$ Soft Condensed Matter, Debye Institute for Nanomaterials Science, Utrecht University, Princetonplein 5, Utrecht 3584 CC, The Netherlands. ${ }^{3}$ Consiglio Nazionale Ricerche-Istituto Sistemi Complessi, UoS Sapienza, P.le A. Moro 2, I-00185 Roma, Italy. *e-mail: f.smallenburg@gmail.com 


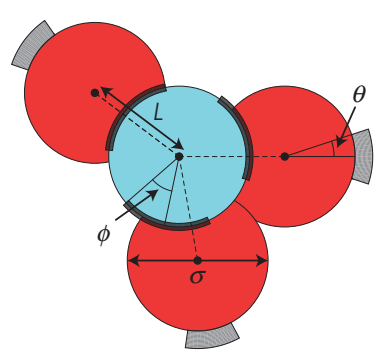

b

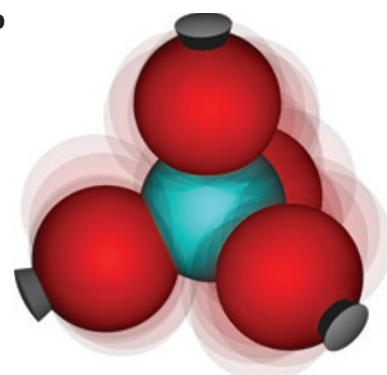

Figure 1 | Tetramer model. Each tetramer consists of five hard spheres of diameter $\sigma$ : a core at the centre and four 'arms' oriented along a tetrahedral geometry. The centres of the arms are located a distance $L$ from the centre of the core. The vector connecting the centre of the core and each arm is allowed to fluctuate within a variable angle $\phi$ from its ideal tetrahedral position. In addition, there is an attractive patch on the surface of each arm characterized by an angular width $\cos \theta=0.95$ and a bond range $\delta=0.251 \sigma$, pointing directly away from the core. $\mathbf{a}$, A two-dimensional schematic diagram of the model, showing a central sphere with three (out of four) arms, indicating the relevant angles and distances. $\mathbf{b}$, The average configuration (solid) and typical fluctuations (partly transparent) for a tetramer with $\cos \phi=0.9$

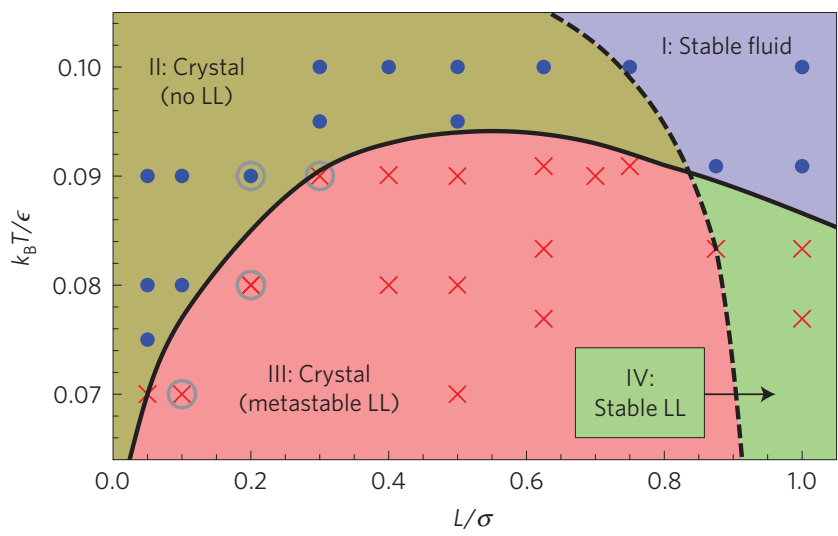

Figure 2 | Conditions for liquid-liquid (LL) phase separation: arm length Diagram showing the region in which $L L$ phase separation and crystallization occur as a function of arm length $L$ and temperture $T$ in units of the bonding energy $(\epsilon)$ for $\cos \phi=0.9$. The state points where LL phase separation was observed are denoted by red crosses; the blue dots indicate points where this was not the case. The solid line indicates the LL critical temperature $T_{\mathrm{c}}$ as determined from the successive umbrella sampling simulations. The dashed line shows the equilibrium crystallization temperature $T_{\mathrm{x}}$ at $\rho_{\mathrm{c}}$, obtained from free-energy calculations performed for $L / \sigma=0.5,0.625,0.75,0.8375$ and 1 . The grey circles indicate spontaneous crystallization into a body-centred cubic crystal in the density region sampled by the LL critical fluctuations.

hundred different densities to provide an accurate description of the thermodynamic behaviour (Methods).

First, we investigate the role of $L$ at fixed arm flexibility. At each state point, we record whether a LL phase separation occurs, and whether the system crystallizes spontaneously during the simulations. Typically, we very clearly observe a LLCP at sufficiently low $T$. The critical number density $\rho_{c}(L)$ is found to be approximately the same if measured in units of the typical distance between the centres of two bonded particles $\sigma+L+\delta / 2$, that is $\rho_{c}^{*}=\rho_{c}(L)(\sigma+L+\delta / 2)^{3} \approx 0.97$. Thus, the free volume at $\rho_{c}$ increases as a function of $L$, affecting both the bonding probability at a given $T$ and the ability of the system to form an interpenetrating

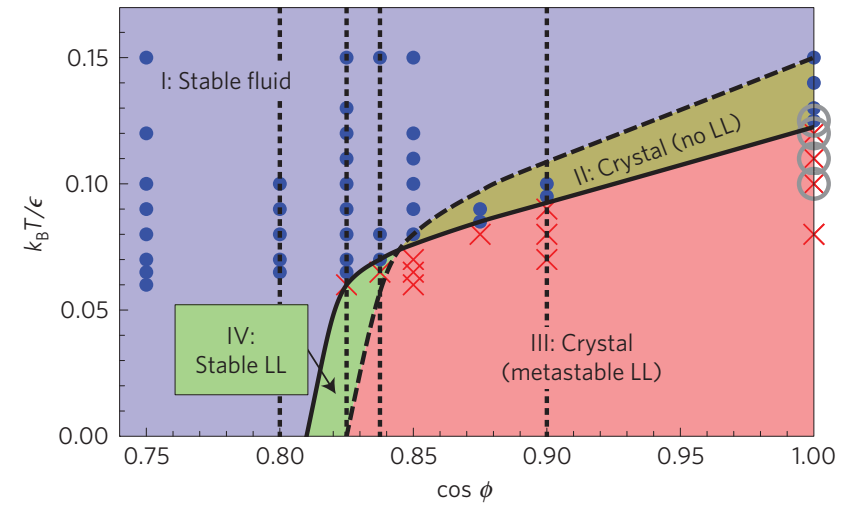

Figure 3 | Conditions for liquid-liquid (LL) phase separation: bond flexibility. Diagram showing the phase behaviour at $\rho_{\mathrm{c}}$ (the LL critical density) in the bond flexibility $(\cos \phi)$ versus temperature $(T)$ plane for $L=0.5 \sigma$. The indicated regions are the same as those shown in Fig. 2 . The red crosses and blue dots indicate points where LL phase separation was or was not detected in the successive umbrella sampling simulations; the grey circles indicate spontaneous crystallization. The dashed and solid black lines are based on free-energy calculations performed at $\cos \phi=0.8,0.825$, $0.8375,0.85,0.875,0.9$ and 1 . The four vertical dotted lines denote the bond flexibility values for which the phase diagrams in Fig. 4 are drawn. $\epsilon$ is the bonding energy.

network. As a result, the corresponding LL critical temperature $T_{c}$ depends significantly on the arm length. The results, summarized in Fig. 2, show a non-monotonous dependence of $T_{c}$ (solid line) on the arm length. For $L \rightarrow 0$, the high packing fraction of the system near the LLCP hinders phase separation, as the hard cores of the particles make it difficult to compress part of the system into a denser liquid phase. In fact, it seems that in our model, a small amount of softness $(L>0)$ is necessary to observe a $L L$ transition at finite $T$. At $L \approx 0.5 \sigma$, there is a maximum in $T_{c}$ beyond which the LL transition is shifted to smaller $T$. This is a trivial consequence of the decrease in $\rho_{c} \sigma^{3}$ as a function of $L$; that is, the bonds required for phase separation at larger $L$ are formed at lower $T$ because increasing the volume per particle disfavours bond formation.

Figure 2 also shows the equilibrium crystallization temperature $T_{\mathrm{x}}(L)$ along the critical isochore $\rho_{\mathrm{c}}$. This line intersects the LL critical-point line $T_{\mathrm{c}}(L)$ around $L=0.82 \sigma$. The two lines separate the parameter space into four regions with different phase behaviour: the standard liquid region, present for all $L$ at large $T$ (denoted by I in Fig. 2); the region where the system crystallizes and there is no LL separation (II); the region where a LL phase separation exists, but is metastable with respect to crystallization (III); and the region where the LL is truly thermodynamically stable (IV).

We note that for small $L$ we also observe spontaneous crystallization into a body-centred cubic (bcc) crystal near the LLCP. This is an effect of the large packing fraction sampled by the critical fluctuations near the LL phase transition, which favours the formation of the closely packed bcc crystal. As expected for this bond flexibility $(\cos \phi=0.9)$, we never observe spontaneous crystallization of the lower density diamond crystal ${ }^{20,25}$. For comparison, we note that the typical (low-pressure) densities of diamond and bcc, when measured in the same reduced units as the critical density, are $\rho_{\text {diamond }}^{*} \simeq 0.7$ and $\rho_{\text {bcc }}^{*} \simeq 1.4$, respectively.

Next, we turn our attention to the effect of arm flexibility on the LL phase transition. Figure 3 shows that increasing the flexibility of the arms (that is, decreasing $\cos \phi)$ lowers $T_{c}(\cos \phi)$. Stiff bonds thus also favour LL phase separation in addition to crystal stability ${ }^{20,25}$, explaining the difficulty in observing a stable LLCP in atomic and molecular network-forming systems, where interactions (for example, hydrogen bonds in water or $s p^{3}$ electronic orbitals in 

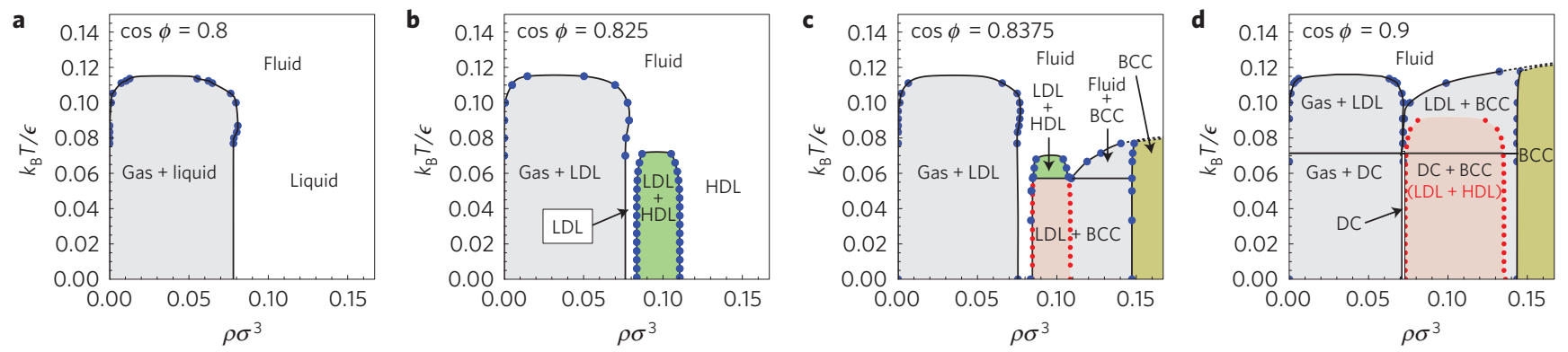

Figure 4 | Effects of bond flexibility on the phase behaviour. a-d, Phase diagrams for different values of the bond flexibility cos $\phi$, for $L=0.5 \sigma$. Symbols indicate state points where phase coexistences were calculated, with red symbols denoting metastable phase coexistences. The green regions indicate a

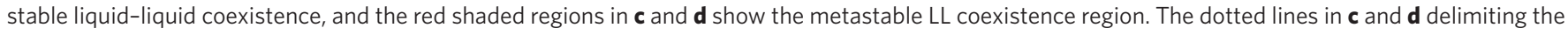
body-centred cubic (bcc) region at high $T$ indicate the expected behaviour of the fluid-bcc coexistence. Crystal structures with a higher density than the

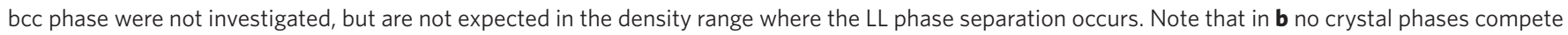
with the LL phase separation, at any temperature. LDL: low-density liquid. HDL: high-density liquid. DC: diamond cubic.

silicon) are highly directional ${ }^{27}$. As in Fig. 2, the equilibrium crystallization temperature $T_{\mathrm{x}}$ intersects $T_{\mathrm{c}}$, around $\cos \phi=0.85$, dividing the diagram into four regions. Again, we find a region (III) where the LL is metastable with respect to crystallization, as proposed for the case of water-like systems, and a region (IV) where the LL is truly thermodynamically stable. In this region, the LL critical point can be experimentally accessed, without any fear of crystallization.

To better elucidate the effect of the arm flexibility on the topology of the phase diagram, we calculate the full phase diagrams, including ordered and disordered phases, in the $T-\rho$ plane for four different values of $\cos \phi$ at fixed $L$. The different panels in Fig. 4 show the progression in the topology of the phase diagram on increasing flexibility. For highly flexible bonds, (Fig. $4 \mathrm{a}, \cos \phi=0.8$ ), we observe a liquid-gas but no LL phase separation. In addition, there is a wide region of densities where the liquid is the stable phase for all $T$. For $\cos \phi=0.825$ (Fig. 4b), we observe both gas-liquid and LL critical points, but still no stable bcc or diamond crystal phase. This is the main finding of this work: the evidence that there are cases in which the LL transition can be studied without the interference of crystallization at any $T$. For $\cos \phi=0.8375$ (Fig. 4c), we still observe both the gas-liquid and the LL critical point. However, a triple point between a low-density liquid (LDL), high-density liquid (HDL) and bcc now exists: the LL phase separation becomes metastable with respect to a LDL-bcc phase separation at $k_{\mathrm{B}} T / \epsilon \approx 0.057$. We note that in all cases, the gas-liquid and LL phase separations occur at distinctly separated chemical potentials: a gas-HDL coexistence never occurs. Finally, for $\cos \phi=0.9$ (Fig. 4d), the LL phase separation becomes fully metastable with respect to crystallization. In addition, as a result of the strong bond directionality, the diamond phase becomes stable at intermediate density and low $T$ (ref. 20). Interestingly, for all $\cos \phi$ values, the liquid side of the liquid-gas coexistence line 'bulges out' to higher densities, analogous to the phase diagram of water ${ }^{28}$.

Several important results stem from the extensive study reported in this Letter. In addition to the identification of two important control parameters (softness and flexibility), our study demonstrates the generality of the LL phenomenon in tetrahedral particles. Beyond a minimum flexibility $(\cos \phi)$ and a minimum softness $(L)$ all models show a genuine LL critical point. Our study also shows that both the LL transition and crystallization are favoured by bond directionality, which destabilizes the homogeneous fluid at densities around $\rho_{c}$ by constraining the topology of the bonding pattern. As shown in Fig. 3, for short bonds and highly directional interactions (that is, for flexibility values typical of atomic and molecular systems) the competition between these phenomena is inevitably won by crystallization. This is the region of $\phi$ and $L$ parameters typical of water, silica and silicon, and explains the exquisite sensitivity of the numerical results to the model parameters ${ }^{29}$. Variations in excluded-volume effects and/or directionality can significantly promote crystallization and suppress the possibility of approaching the LL critical point under metastable conditions. As shown in Figs 3 and 4, our calculations confirm that both for $L \rightarrow 0$ and $\cos \phi \rightarrow 1$ (the values appropriate for the case of water, see ref. 27 and Supplementary Information), spontaneous crystallization prevails, consistent with experimental results. Nonetheless, our simple model shows that, depending on two key parameters, a LL phase transition is a general feature of tetrahedral liquids that has to be considered when studying such systems. Moreover, as speculated a long time ago in the case of water, the pattern of anomalies departing from the critical point affects the behaviour of the system even in the region where the fluid is stable ${ }^{8}$. These anomalies, for example, extrema in the density, isobaric heat capacity, and isothermal compressibility, occur in the present model as well, and are discussed in the Supplementary Information.

Finally, we have shown that sufficiently flexible bonds and sufficient softness give rise to a window where the full LL coexistence is thermodynamically stable. Interestingly, this range of flexibilities is within reach in the soft-matter realm. Patchy particles where the number and position of patches is fully controllable (including tetrahedral geometry) $)^{21-23}$ are nowadays experimentally realizable. These advances open up the possibility of synthesizing colloidal particles with the finely tuned softness and bonding flexibility required to experimentally explore the LLCP in the absence of crystallization. The new generation of patchy colloids may well provide the long-sought experimental evidence that a singlecomponent liquid can phase separate into two distinct liquids.

\section{Methods}

In our model, each particle consists of a core sphere with four tetrahedrally oriented arms (Fig. 1). The central sphere and the arms are modelled as interpenetrating hard spheres with diameter $\sigma$, with the centre of each arm located a distance $L$ from the centre of the core. On the surface of each arm is a single attractive patch, pointing directly away from the central core. The patch is modelled, using a standard Kern-Frenkel potential ${ }^{24}$, as a square-well type attraction of depth $\epsilon$ : two particles are either bound or unbound, with no intermediate energy levels. The opening angle of the patch is given by $\cos \theta=0.95$, and the maximum interaction range $\delta=0.251 \sigma$. This choice ensures that each patch can form a bond with only a single other patch. Furthermore, to model arm flexibility, each arm can freely rotate around the core sphere within a maximum angle $\phi$ from its ideal (tetrahedral) position. Note that there are no hard-core interactions within a particle: spheres belonging to the same tetramer can overlap.

All simulations were performed following a Monte Carlo scheme. Each Monte Carlo simulation included translation moves, rotation moves rotating a full tetramer, rotation moves rotating only the core sphere, and rotation moves 
that rotate a single arm around the core. In addition, we added volume moves for isobaric simulations, and particle insertion and deletion moves for grand-canonical SUS (ref. 26) simulations.

To detect LL phase separation, we used SUS simulations, generating the relative free energy as a function of the density ${ }^{26}$. To calculate the phase diagrams in Fig. 4, and the crystallization lines in Figs 2 and 3, we performed free-energy calculations using standard thermodynamic integration techniques. For the liquid phases, we used a high-temperature fluid as a reference state, where we obtained the Helmholtz free energy $F$ by combining the chemical potential $\mu$ (evaluated from a SUS simulation) and the pressure $P$ at the same density $\rho$ (taken from an NPT Monte Carlo simulation), using:

$$
f \equiv F / N=\mu-P / \rho
$$

where $N$ is the number of particles. We then used thermodynamic integration by integrating the temperature along an isochore:

$$
\beta_{2} f\left(\rho, T_{2}\right)=\beta_{1} f\left(\rho, T_{1}\right)+\int_{\beta_{1}}^{\beta_{2}} \mathrm{~d} \beta u(\rho, T)
$$

with $u(\rho, T)$ being the average potential energy per particle, $\beta=1 / k_{\mathrm{B}} T$ and $k_{\mathrm{B}}$ being Boltzmann's constant. Typically, at sufficiently low temperatures ${ }^{20}$, the energy as a function of temperature takes the form

$$
u / \epsilon=-2+c \exp (-\beta \epsilon / 2)
$$

where -2 is the ground-state energy (where each particle has four bonds) and $c$ is a (density-dependent) constant. This allows for straightforward extrapolation of the free energies to arbitrarily low temperatures after the regime in equation (1) is reached. This regime is accessible in our simulations for all cases where the fluid does not crystallize at low $T$ (that is, $\cos \phi \lesssim 0.9$ ). System sizes for the SUS simulations were chosen such that near the LL transition the system contained $N \approx 150$ particles. Other free-energy calculations were performed with similar or larger $N$.

The investigated crystal structures were diamond and bcc (corresponding to ice $\mathrm{I}_{c}$ and ice VII in water, respectively). Both are fully bonded structures and as such have the (same) lowest possible potential energy. Depending on $\cos \phi$ and $L$, there will be a variety of other fully bonded crystal structures. However, the structures commensurate with an ideal tetrahedral geometry will always have the highest entropy and hence will be more stable at the densities where they are accessible. Thus, the only crystals that might compete with the LL phase separation are diamond and bcc. At high density (that is, beyond the bcc density), the stable crystalline phases will be controlled by a competition between packing, energy and entropy, but as these phases will not change the stability of the LL phase transition, they are outside the scope of this Letter. Similarly, we do not expect these crystal phases to affect the phase diagrams in Fig. 4, with the possible exception of the (dashed) high-density liquid-bcc lines in Fig. 4c,d.

To determine free energies for the crystal phases, we used an Einstein crystal $\operatorname{approach}^{30}$, for each $T$ where a phase coexistence was calculated. Subsequently, using NPT Monte Carlo simulations, we employed thermodynamic integration along the density at constant temperature:

$$
\beta f(\rho, T)=\beta f\left(\rho_{\text {ref }}, T\right)+\int_{\rho_{\text {ref }}}^{\rho} \mathrm{d}\left(\rho^{\prime}\right)\left\langle\frac{\beta P}{\rho^{\prime 2}}\right\rangle_{\rho^{\prime}}
$$

where $\rho_{\text {ref }}$ is the density where the reference free energy was calculated. Phase coexistences were determined using common tangent constructions (see Supplementary Fig. 4 for examples).

To determine whether spontaneous crystallization occurred in our SUS simulations, we used a bond-order parameter to find the largest crystalline cluster in the system. First, we determine the neighbours of each particle using a solid-angle-based nearest-neighbour method ${ }^{31}$. We then calculate for each particle the complex vector $\mathbf{q}_{l}$, the expansion of its environment in terms of spherical harmonics $Y_{l m}$, with $l$ being the order of the symmetry of the crystal, and $-l \leq m \leq l$ :

$$
q_{l}^{m}(i)=\frac{1}{N_{b}(i)} \sum_{j=1}^{N_{b}} Y_{l m}\left(\hat{\mathbf{r}}_{i j}\right)
$$

Here, $N_{b}$ is the number of neighbours of particle $i$, and $\hat{\mathbf{r}}_{i j}$ is the normalized vector connecting particle $i$ to particle $j$. For the purpose of finding crystalline clusters, two particles are considered bonded when their environments are sufficiently similar. To determine this, we calculate:

$$
d_{l}(i, j)=\operatorname{Re}\left(\frac{\mathbf{q}_{l}(i) \cdot \mathbf{q}_{l}^{*}(j)}{\left|\mathbf{q}_{l}(i)\right|\left|\mathbf{q}_{l}(j)\right|}\right)
$$

To detect bcc clusters, we choose $l=6$, consider two particles bonded if $d_{l}(i, j)>0.6$, and label any particle that has at least 5 bonds as a crystalline particle. For diamond, two particles are considered bonded if either $d_{l}(i, j)<-0.87$ or $-0.3<d_{l}(i, j)<0.1$ (ref. 25 ), and only particles with 4 bonds are considered crystalline. Systems were considered to have crystallized spontaneously when more than $10 \%$ of the system was crystalline. Note that although the $q_{6}$-based order parameter is sensitive to multiple crystal structures, visual inspection of the detected clusters consistently showed a bcc structure in all cases.

Received 19 February 2014; accepted 13 June 2014; published online 27 July 2014

\section{References}

1. Poole, P. H., Sciortino, F., Essmann, U. \& Stanley, H. E. Phase behaviour of metastable water. Nature 360, 324-328 (1992).

2. Mishima, O. \& Stanley, H. E. The relationship between liquid, supercooled and glassy water. Nature 396, 329-335 (1998).

3. Mishima, O., Calvert, L. \& Whalley, E. An apparently first-order transition between two amorphous phases of ice induced by pressure. Nature 314, 76-78 (1985)

4. Amann-Winkel, K. et al. Water's second glass transition. Proc. Natl Acad. Sci. USA 110, 17720-17725 (2013)

5. Vasisht, V. V., Saw, S. \& Sastry, S. Liquid-liquid critical point in supercooled silicon. Nature Phys. 7, 549-553 (2011).

6. Glosli, J. N. \& Ree, F. H. Liquid-liquid phase transformation in carbon. Phys. Rev. Lett. 82, 4659-4662 (1999).

7. Debenedetti, P. G. Supercooled and glassy water. J. Phys. Condens. Matter 15, R1669-R1726 (2003)

8. Holten, V., Bertrand, C. E., Anisimov, M. A. \& Sengers, J. V. Thermodynamics of supercooled water. J. Chem. Phys. 136, 094507 (2012).

9. Taschin, A., Bartolini, P., Eramo, R., Righini, R. \& Torre, R. Evidence of two distinct local structures of water from ambient to supercooled conditions. Nature Commun. 4, 2401 (2013).

10. Beye, M., Sorgenfrei, F., Schlotter, W. F., Wurth, W. \& Föhlisch, A. The liquid-liquid phase transition in silicon revealed by snapshots of valence electrons. Proc. Natl Acad. Sci. USA 107, 16772-16776 (2010).

11. Liu, Y., Panagiotopoulos, A. Z. \& Debenedetti, P. G. Low-temperature fluid-phase behavior of ST2 water. J. Chem. Phys. 131, 104508 (2009).

12. Tu, Y., Buldyrev, S. V., Liu, Z., Fang, H. \& Stanley, H. E. Different water scenarios for a primitive model with two types of hydrogen bonds. Europhys. Lett. 97, 56005 (2012).

13. Abascal, J. L. \& Vega, C. Widom line and the liquid-liquid critical point for the tip4p/2005 water model. J. Chem. Phys. 133, 234502 (2010).

14. Corradini, D., Rovere, M. \& Gallo, P. A route to explain water anomalies from results on an aqueous solution of salt. J. Chem. Phys. 132, 134508 (2010).

15. Poole, P. H., Bowles, R. K., Saika-Voivod, I. \& Sciortino, F. Free energy surface of ST2 water near the liquid-liquid phase transition. J. Chem. Phys. 138, 034505 (2013)

16. Liu, Y., Palmer, J. C., Panagiotopoulos, A. Z. \& Debenedetti, P. G. Liquid-liquid transition in ST2 water. J. Chem. Phys. 137, 214505 (2012).

17. Limmer, D. T. \& Chandler, D. The putative liquid-liquid transition is a liquid-solid transition in atomistic models of water. J. Chem. Phys. 135, 134503 (2011).

18. Kesselring, T. A., Franzese, G., Buldyrev, S. V., Herrmann, H. J. \& Stanley, H. E. Nanoscale dynamics of phase flipping in water near its hypothesized liquid-liquid critical point. Sci. Rep. 2 (2012).

19. Hsu, C. W., Largo, J., Sciortino, F. \& Starr, F. W. Hierarchies of networked phases induced by multiple liquid-liquid critical points. Proc. Natl Acad. Sci. USA 105, 13711-13715 (2008)

20. Smallenburg, F. \& Sciortino, F. Liquids more stable than crystals in particles with limited valence and flexible bonds. Nature Phys. 9, 554-558 (2013).

21. Li, Y. et al. Controlled assembly of dendrimer-like DNA. Nature Phys. 3, $38-42$ (2003).

22. Roh, Y. H., Ruiz, R. C., Peng, S., Lee, J. B. \& Luo, D. Engineering DNA-based functional materials. Chem. Soc. Rev. 40, 5730-5744 (2011).

23. Biffi, S. et al. Phase behavior and critical activated dynamics of limited-valence DNA nanostars. Proc. Natl Acad. Sci. USA 110, 15633-15637 (2013). 
24. Kern, N. \& Frenkel, D. Fluid-fluid coexistence in colloidal systems with short-ranged strongly directional attraction. J. Chem. Phys. 118, 9882-9889 (2003).

25. Romano, F., Sanz, E. \& Sciortino, F. Crystallization of tetrahedral patchy particles in silico. J. Chem. Phys. 134, 174502 (2011).

26. Virnau, P. \& Müller, M. Calculation of free energy through successive umbrella sampling. J. Chem. Phys. 120, 10925 (2004).

27. Saika-Voivod, I., Smallenburg, F. \& Sciortino, F. Understanding tetrahedral liquids through patchy colloids. J. Chem. Phys. 139, 234901 (2013).

28. Haar, L., Gallagher, J. \& Kell, G. NBS/NRC Steam Tables (Hemisphere, 1985).

29. Moore, E. B. \& Molinero, V. Structural transformation in supercooled water controls the crystallization rate of ice. Nature 479, 506-508 (2011).

30. Vega, C., Sanz, E., Abascal, J. \& Noya, E. Determination of phase diagrams via computer simulation: Methodology and applications to water, electrolytes and proteins. J. Phys. Condens. Matter 20, 153101 (2008).

31. van Meel, J. A., Filion, L., Valeriani, C. \& Frenkel, D. A parameter-free, solid-angle based, nearest-neighbor algorithm. J. Chem. Phys. 136, 234107 (2012).

\section{Acknowledgements}

F.Sciortino and F.Smallenburg acknowledge support from

ERC-226207-PATCHYCOLLOIDS and MIUR-PRIN. L.F. acknowledges

financial support from the Dutch Sector Plan Physics and Chemistry, and an NWO-Veni grant.

\section{Author contributions}

F.Sciortino suggested the study. F.Sciortino, L.F. and F.Smallenburg designed the research, performed the simulations and the data analysis, contributed to the interpretation of the results and wrote the paper.

\section{Additional information}

Supplementary information is available in the online version of the paper. Reprints and permissions information is available online at www.nature.com/reprints.

Correspondence and requests for materials should be addressed to F.Smallenburg.

\section{Competing financial interests}

The authors declare no competing financial interests. 